\title{
NUSTAR observations of the supergiant X-ray pulsar IGR J18027-2016: accretion from the stellar wind and possible cyclotron absorption line
}

\author{
Alexander A. Lutovinov ${ }^{1,2 \star}$, Sergey S. Tsygankov ${ }^{3}$, Konstantin A. Postnov ${ }^{4}$, \\ Roman A. Krivonos ${ }^{1}$, Sergey V. Molkov ${ }^{1}$ and John A. Tomsick ${ }^{5}$ \\ 1 Space Research Institute of the Russian Academy of Sciences, Profsoyuznaya str. 84/32, Moscow, 117997, Russia \\ ${ }^{2}$ Moscow Institute of Physics and Technology, Institutskiy per. 9, Dolgoprudny, Moscow Region, 141700, Russia \\ 3 Tuorla Observatory, Department of Physics and Astronomy, University of Turku, Väisäläntie 20, FI-21500 Piikkiö, Finland \\ ${ }^{4}$ Moscow M.V. Lomonosov State University, Sternberg Astronomical Institute, 119992 Moscow, Russia \\ 5 Space Sciences Laboratory, 7 Gauss Way, University of California, Berkeley, CA 94720-7450, USA
}

Accepted .... Received ...

\begin{abstract}
We report on the first focused hard X-ray view of the absorbed supergiant system IGR J18027-2016 performed with the NuSTAR observatory. The pulsations are clearly detected with a period of $P_{\text {spin }}=139.866(1) \mathrm{s}$ and a pulse fraction of about $50-60 \%$ at energies from 3 to $80 \mathrm{keV}$. The source demonstrates an approximately constant X-ray luminosity on a time scale of more than dozen years with an average spin-down rate of $\dot{P} \simeq 6 \times 10^{-10} \mathrm{~s} \mathrm{~s}^{-1}$. This behaviour of the pulsar can be explained in terms of the wind accretion model in the settling regime. The detailed spectral analysis at energies above $10 \mathrm{keV}$ was performed for the first time and revealed a possible cyclotron absorption feature at energy $\sim 23 \mathrm{keV}$. This energy corresponds to the magnetic field $B \simeq 3 \times 10^{12}$ $\mathrm{G}$ at the surface of the neutron star, which is typical for X-ray pulsars.
\end{abstract}

Key words: accretion, accretion discs - magnetic fields - stars: individual: IGR J18027-2016 - X-rays: binaries.

\section{INTRODUCTION}

IGR J18027-2016 was discovered by the INTEGRAL observatory during deep observations of the Galactic Center region as a faint persistent hard X-ray source (Revnivtsev et al. 2004). Follow-up observations performed with the XMM-Newton observatory in soft X-rays allowed for an improvement in the source localization, enabling nearinfrared observations with the NTT/ESO telescope and establishing the nature of its normal companion as a supergiant star of B1Ib type (Torrejón et al. 2010) at the distance of $12.4 \mathrm{kpc}$ (see also Mason et al. 2011, for slightly different type).

Using the archival data of the BeppoSAX observatory Augello et al. (2003) revealed that the serendipitous source SAX J1802.7-2017 is spatially associated with IGR J180272016. These authors showed that this source is an X-ray pulsar with a spin period of $139.6 \mathrm{~s}$, residing in a binary system with the orbital period of 4.6 days. Later the orbital parameters of the system were improved by Hill et al. (2005) and Falanga et al. (2015).

* E-mail: aal@iki.rssi.ru
The average spectrum of IGR J18027-2016, measured in a wide energy band (Lutovinov et al. 2005), demonstrates a cutoff at high energies, which is typical for X-ray pulsars (see, e.g., Filippova et al. 2005). In addition, a relatively high absorption value, $N_{\mathrm{H}}=6.8 \times 10^{22} \mathrm{~cm}^{-2}$, was revealed at low energies (Hill et al. 2005), and the source was classified as an absorbed binary system with a supergiant companion (Walter et al. 2015). No search for a cyclotron absorption line and no pulse phase resolved spectroscopic study for IGR J18027-2016 has been performed to date.

In this paper we report results of observations of IGR J18027-2016, collected with the NuSTAR observatory and Swift/XRT telescope in Aug 2015. The main purpose of these observations was to reconstruct the source broadband spectrum with high accuracy and to search for a cyclotron absorption line.

\section{OBSERVATIONS AND DATA REDUCTION}

The Nuclear Spectroscopic Telescope Array (NuSTAR) has two identical X-ray telescope modules, each equipped with independent mirror systems and focal plane detector units, 


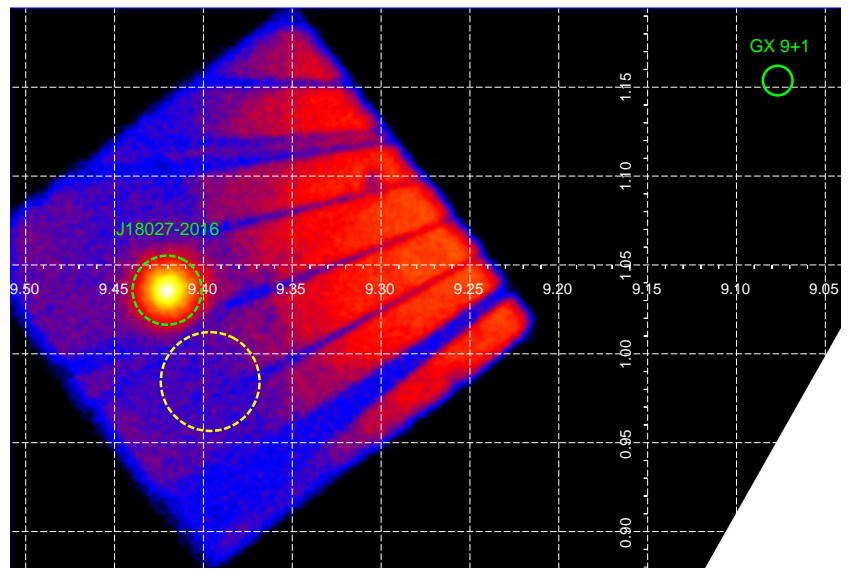

Figure 1. The NUSTAR image of IGR J18027-2016 in the $3-$ $79 \mathrm{keV}$ energy band obtained with FPMA module (the picture for another module FPMB is practically identical). Green and yellow dashed circles denote source and background extraction regions, respectively. The ghost-ray (GR) contamination is revealed by a characteristic radial streaks pattern in the image. The GR photons originate from GX 9+1, whose position is marked by the solid green circle. The white grid indicates the Galactic coordinate system in degrees.

also referred to as FPMA and FPMB (Harrison et al. 2013). The unique multilayered coating of the grazing-incidence Wolter-I design optics provides X-ray imaging in the energy range $3-79 \mathrm{keV}$ with an angular resolution of $18^{\prime \prime}$ (FWHM). Each telescope has a field-of-view (FOV) of $\sim 13^{\prime} \times 13^{\prime}$. CdZnTe pixel detectors of FMPA and FPMB provide spectral resolution of $400 \mathrm{eV}$ (FWHM) at $10 \mathrm{keV}$.

IGR J18027-2016 was observed with NuSTAR on August 27, 2015 (MJD 57261.0141 - 57261.9922, ObsID 30101049002) during Cycle 1 of the Guest Observer Program with a total on-target exposure of $\sim 36 \mathrm{ks}(\sim 32.6 \mathrm{ks}$ after the dead-time correction and GX 9+1 flare removal, see below). The presence of bright nearby sources can contaminate the NuSTAR observations due to the aperture stray light - unfocused X-rays reaching the NuSTAR detectors. The contamination that we see is often called ghost-rays (GR), which are photons that are reflected from only upper or lower X-ray mirror sections (so-called single scattering photons, see, e.g., Wik et al. 2014; Krivonos et al. 2014; Mori et al. 2015). In a close proximity to IGR J18027-2016 (at the angular distance of $21.5^{\prime}$ ), the bright low-mass X-ray binary system GX $9+1$ is located. Therefore, we estimated initially (at the observation planning stage) a possible contamination of GR photons from this source to the useful signal. According to NuSTAR's experience with GRs from the very bright system $4 \mathrm{U} 1630-47$ (Bodaghee et al. 2014), the direct GRs are observed within $\mathrm{a} \sim 20^{\prime}$ radius circle, which made observations of IGR J18027-2016 possible. Indeed, Fig. 1 demonstrates that there is no a strong GR contamination from the persistent emission of GX $9+1$ at the position of IGR J18027-2016. However, a strong X-ray flare was detected in the light curve of IGR J18027-2016. Timing and spectral characteristics of this event are typical for Type I X-ray bursts observed from some accreting neutron stars. Therefore, we suggest that this was a burst from GX $9+1$,

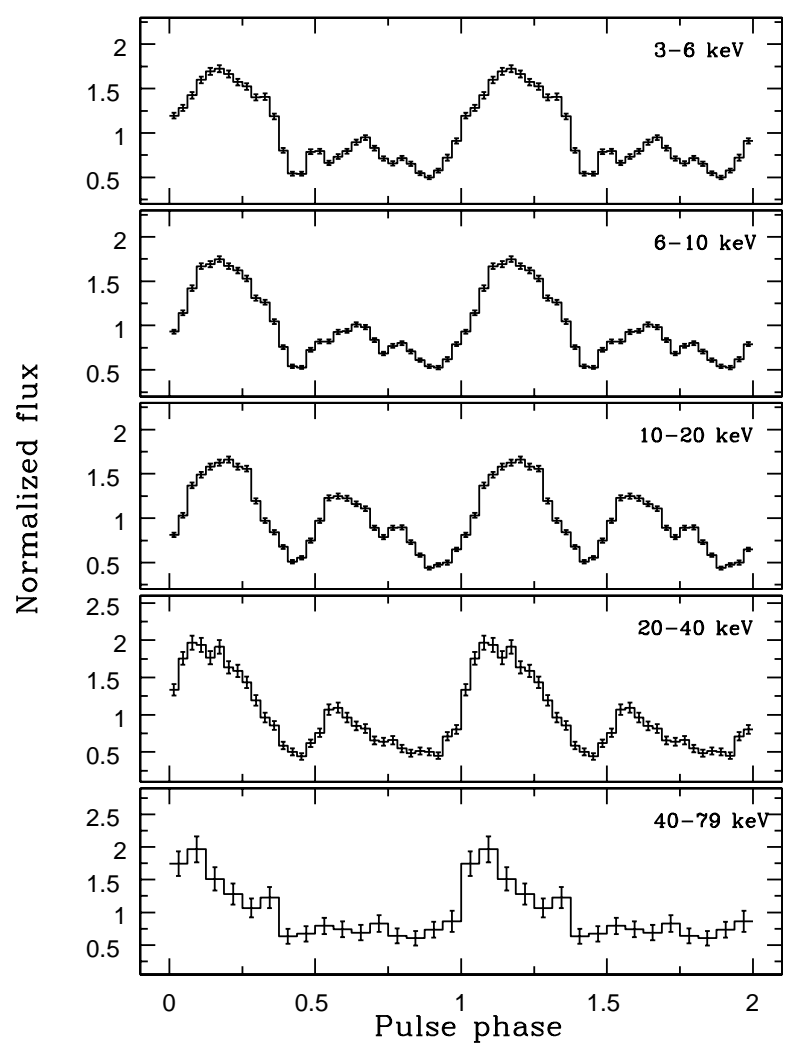

Figure 2. Pulse profiles of IGR in five energy bands 3-6, 6-10, 10-20, 20-40, and 40-79 keV (from top to bottom). The profiles are normalized by the mean flux in each band and plotted twice for clarity.

and we removed the corresponding part of the data from the following analysis.

We processed the raw observational data to produce cleaned event files for the FPMA and FPMB modules using the standard NuSTAR Data Analysis Software (NuSTARDAS) v1.6.0 provided under HEASOFT v6.19 with CALDB version 20160502. Using the NUPRODUCTS routine, we extracted source counts for spectral and timing analysis within $70^{\prime \prime}$ of the centroid position (dashed green circle in Fig. 1), which corresponds to $\sim 80 \%$ of the enclosed PSF fraction (Madsen et al. 2015). Taking into account possible GR contamination, we extracted the background spectrum and light curves in the region with a radius of $100^{\prime \prime}$ (dashed yellow circle), located at nearly same distance from GX 9+1 (its position is indicated by the solid green circle in Fig. 1).

For timing analysis and pulse phase-resolved spectroscopy, the data were corrected for the barycenter of the Solar System with the BARYCORR command and for the binary system motion using orbital parameters from Falanga et al. (2015).

The Swift/XRT instrument observed IGR J18027-2016 simultaneously with the NuSTAR observatory on August 27, 2015 (MJD 57261.6525 - 57261.7159, ObsID 00081660001) with a total exposure of $\sim 2 \mathrm{ks}$. The Swift/XRT data were used to supplement the NUSTAR data in the soft energy 


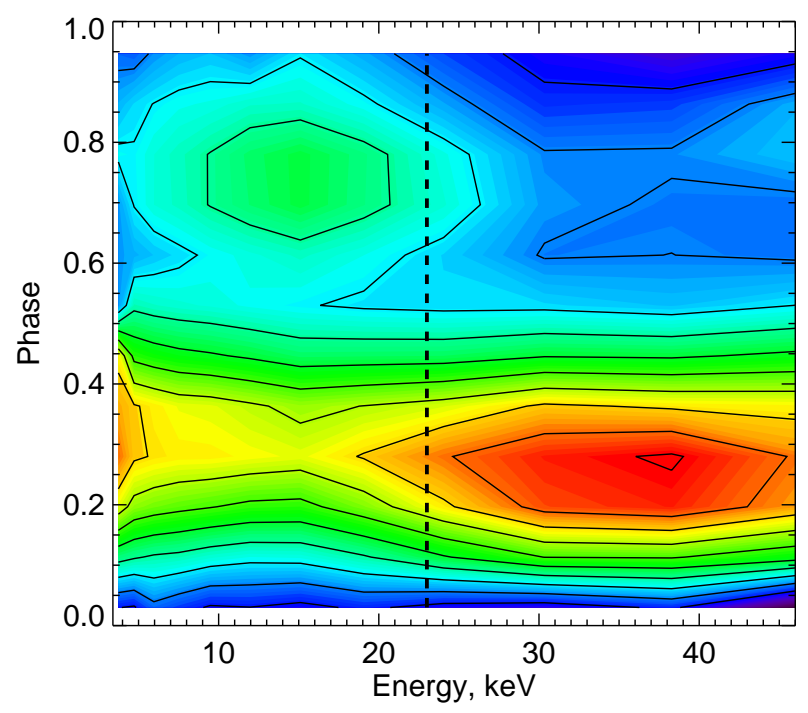

Figure 3. "Energy - pulse phase" distribution of intensity for IGR J18027-2016. Dashed vertical line shows the position of the cyclotron line revealed in the source spectrum. Solid contours represent levels of the equal normalized flux from 0.5 to 1.85 with step of 0.15 .

band and to better determine the level of absorption. These data were reduced using appropriate software ${ }^{1}$.

The final data analysis (timing and spectral) was performed with the FTOOLS 6.7 software package.

\section{TIMING ANALYSIS}

The NuSTAR observatory provides data with very good time and energy resolution, allowing us to investigate temporal properties of IGR J18027-2016 at energies above $10 \mathrm{keV}$ in detail for the first time. In general, the source light curve measured by NuSTAR demonstrates two types of variability on time scales longer than the pulse period and shorter than the orbital period: smooth changes of the source intensity during the observation and relatively quick variations (up to $25-30 \%$ ) on the time scale of several thousand seconds. It can be naturally expected that both types of variations are connected with gradual changes of the stellar wind density over the orbit of the neutron star and its local inhomogeneities.

The pulse period and its uncertainty were determined using the combined light curve from both modules FPMA and FPMB in the wide energy band (3-79 keV) and corrected both for the motion of Earth around Sun and the neutron star in the binary system. To estimate an uncertainty on the pulse period we generated $10^{4}$ trial light curves (using the statistics from the original one) and determined the pulse period value in each of them using the EFSEARCH procedure from FTOOLS package. Finally, the distribution of periods was constructed with the mean and standard deviation corresponding to the proper pulse period and its $1 \sigma$ uncertainty, respectively (see, e.g.,

1 http://swift.gsfc.nasa.gov

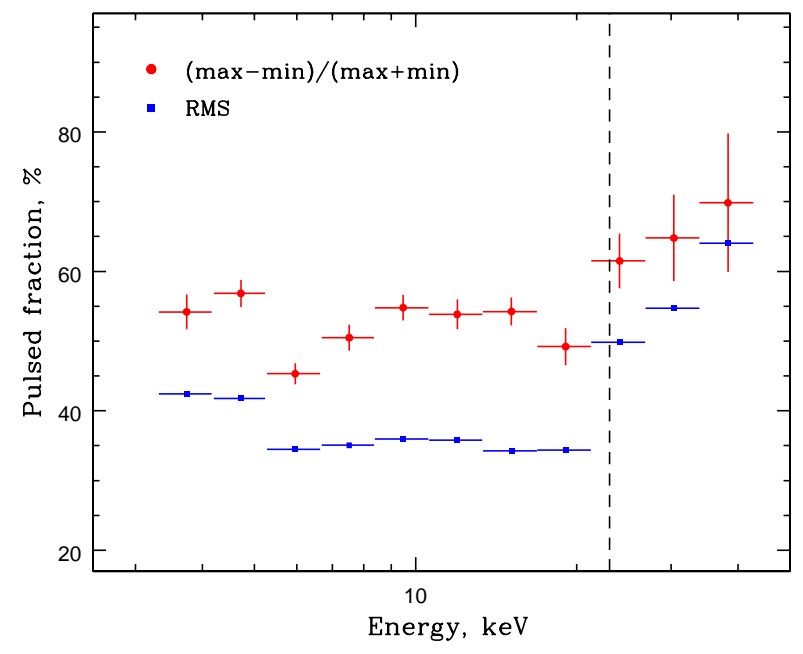

Figure 4. Dependence of the pulsed fraction of IGR J18027-2016 on energy calculated in two different ways (see text for details). The dashed line shows the position of the cyclotron line that may be present in the source spectrum.

Boldin, Tsygankov, \& Lutovinov 2013, for details). The final value of the pulse period, obtained by such an analysis, is $P_{\text {spin }}=139.866(1)$. This value is larger by about $\simeq 0.25 \mathrm{~s}$ compared with the previous measurements done by the BeppoSaX and XMM-Newton observatories (Hill et al. 2005), resulting in the mean spin-down rate of $\dot{P} \simeq 6 \times 10^{-10} \mathrm{~s} \mathrm{~s}^{-1}$.

To produce the pulse profile we used the combined light curves from both modules in order to get better statistics. The pulse profiles in five different energy bands 3-6, 610, 10-20, 20-40, and 40-79 keV (from top to bottom) are shown in Fig. 2. In all energy bands it has a double peak shape with different relative intensities of the peaks.

The evolution of the pulse profile with energy can be illustrated by the "energy - pulse phase" distribution of the intensity constructed with the high energy resolution (see Fig. 3). These pulse profiles were also normalized by the mean flux in each energy band. Details of the technique of the construction of such a 2D-distribution are described by Lutovinov \& Tsygankov (2009) and have already been successfully used in a number of works. From Fig. 3 , it is clear that the second peak has its highest relative intensity between $\sim 10$ and $\sim 20 \mathrm{keV}$. At higher energies the first peak completely dominates the profile. It is important to note that these two energy ranges are divided by the possible cyclotron absorption line revealed in the source spectrum (see Section 4). Its position is shown with the dashed line in Fig. 3.

The pulsed fraction below the cyclotron line energy depends on energy only slightly with a value of $50-60 \%$ (see Fig. 4). At higher energies one can see the gradual increase of the pulsed fraction, typical for the majority of X-ray pulsars (Lutovinov \& Tsygankov 2009).

To avoid possible biases due to the pulse profile peculiarities or statistics we used two different definitions of the pulsed fraction. The "standard" one is $\mathrm{PF}=\left(F_{\max }-\right.$ $\left.F_{\min }\right) /\left(F_{\max }+F_{\min }\right)$, where $F_{\max }$ and $F_{\min }$ are maximum and minimum fluxes in the pulse profile, respectively. Red circles in Fig. 4 show the pulsed fraction defined by this manner. Another way to characterize the pulse profile intensity 


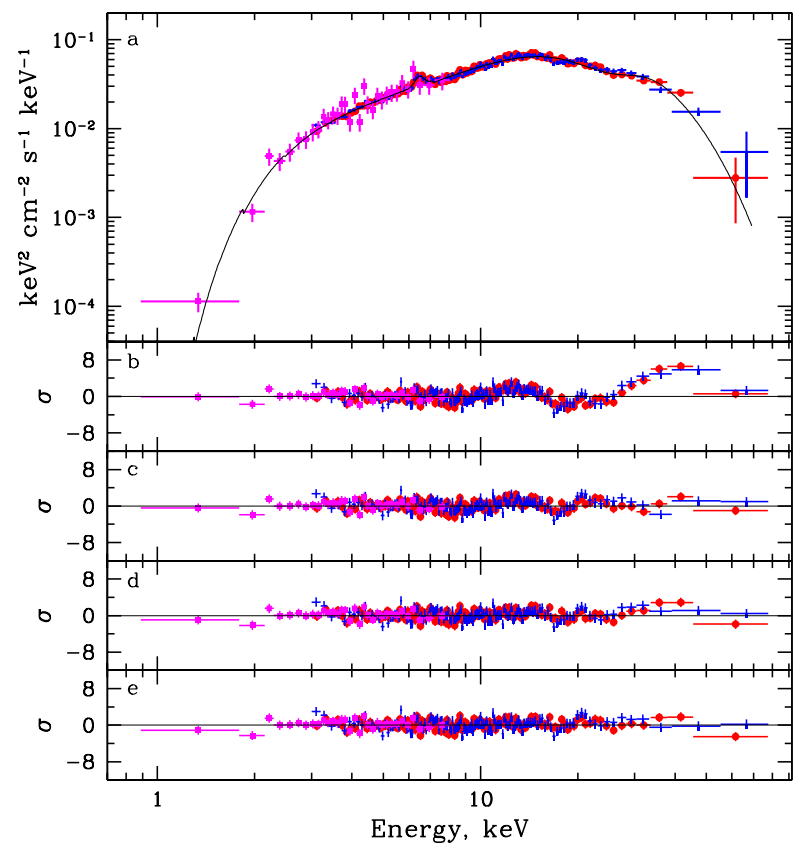

Figure 5. (a) Pulse phase averaged spectrum of IGR J180272016 obtained with the NuSTAR observatory and the Swift/XRT instrument. Red circles and blue crosses correspond to FPMA and FPMB modules data, respectively; magenta points - to the XRT data. The black line shows the best fit model. (b-e) Residuals from different models (see text for details).

variations is to use the relative Root Mean Square (RMS), which can be calculated by the following equation:

$$
R M S=\frac{\left(\frac{1}{N} \sum_{i=1}^{N}\left(P_{i}-\langle P\rangle\right)^{2}\right)^{\frac{1}{2}}}{\langle P\rangle},
$$

where $P_{i}$ is the background-corrected count rate in a given bin of the pulse profile, $\langle P\rangle$ is the profile-averaged count rate, and $N$ is the total number of phase bins in the profile.

The overall behaviour of the pulsed fraction calculated using different approaches agrees very well with the only difference being a slightly lower absolute value for the RMS (blue squares in Fig. 4). This dependence shows two distinct features: the increase of the pulse fraction with energy and its peculiar change at $22-24 \mathrm{keV}$. Note that there is another similar change of the pulsed fraction around 6-7 keV probably connected with the fluorescent iron emission line.

\section{SPECTRAL ANALYSIS}

The working energy range of the NuSTAR observatory (3-79 keV), and its unprecedent sensitivity at $>10 \mathrm{keV}$ makes the observatory an ideal instrument to search for cyclotron resonant scattering features (or, in other words, cyclotron absorption lines) in the spectra of X-ray pulsars (for more recent examples see, e.g., Bodaghee et al. 2016; Tsygankov et al. 2016). Moreover, the quality of the $N u S$ $T A R$ data is so high that the simple theoretical models usually used for the modelling of spectra of X-ray pulsars are often not able to approximate them satisfactorily, and we were

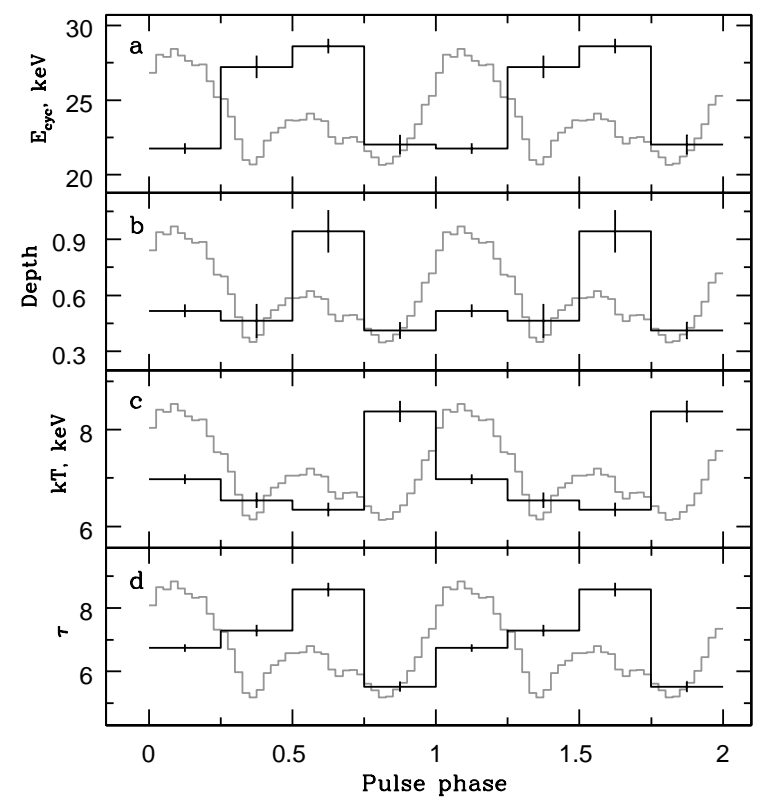

Figure 6. Spectral parameters of the best-fit model as a function of pulse phase. The black histogram in the panels represents: (a) cyclotron line energy, (b) cyclotron line depth, (c) temperature and (d) optical depth in the COMPTT model. The grey line in each panel shows the pulse profile in a wide energy range.

met with this problem in the analysis of the IGR J180272016 spectrum.

As mentioned above, the spectrum of IGR J180272016 is typical for accreting X-ray pulsars. In particular, it demonstrates an exponential cutoff at high energies (Fig. 5a). Therefore, as a first step, we attempted to approximate it with several commonly used models: a power law with an exponential cutoff (CUTOFFPL in the XSPEC package), the NPEX model, the broken power law model (see, e.g. Hill et al. 2005), thermal inverse Comptonization (COMPTT in the XSPEC package), a power law with a high energy cutoff (POWERLAW*HIGHCUT in the XSPEC package, White, Swank, \& Holt 1983). To take into account the absorption in the system, the PHABS model was used with the abundances from Anders \& Grevesse (1989).

The source and background spectra from both FPMA and FPMB modules of NuSTAR as well as from the Swift/XRT telescope were used for simultaneous fitting. To take into account the uncertainty in the instrument calibrations as well as the lack of full simultaneity of observations by NuSTAR and Swift (the first one observed IGR J180272016 for nearly the whole day, while the latter one observed for only $2 \mathrm{ks}$ ), cross-calibration constants between them were included in all spectral models.

None of the models that we used describe the spectrum very well - typically, there are strong deviations at low energies and a depression around $20 \mathrm{keV}$ with a complex shape. Moreover, two models (the broken power law and POWERLAW*HIGHCUT) suffer from the abrupt breaks at the break and cutoff energies, respectively. This can lead to artificial narrow line features around these energies (see, e.g., Shtykovsky et al. 2016). Therefore, in the following analysis, we used the COMPTT model, which we consider to be the most reliable one. 
Table 1. Best-fit parameters of the IGR J18027-2016 spectrum with different models.

\begin{tabular}{|c|c|c|c|c|}
\hline Parameter & Comptt & Comptt+Gabs & PowHigh ${ }^{\mathrm{a}}$ & PowHigh+Gabs \\
\hline$C 1$ & $1.014 \pm 0.006$ & $1.013 \pm 0.006$ & $1.013 \pm 0.006$ & $1.013 \pm 0.006$ \\
\hline$C 2$ & $0.87 \pm 0.04$ & $0.86 \pm 0.04$ & $0.86 \pm 0.04$ & $0.86 \pm 0.04$ \\
\hline$N_{\mathrm{H}}, \times 10^{22} \mathrm{~cm}^{-2}$ & $3.55 \pm 0.30$ & $3.22 \pm 0.30$ & $3.36 \pm 0.22$ & $3.23 \pm 0.21$ \\
\hline$k T_{0}, \mathrm{keV}$ & $0.61 \pm 0.09$ & $0.70 \pm 0.09$ & - & - \\
\hline$k T, \mathrm{keV}$ & $5.91 \pm 0.04$ & $6.86 \pm 0.08$ & - & - \\
\hline$\tau$ & $7.59 \pm 0.13$ & $7.41 \pm 0.14$ & - & - \\
\hline Photon index & - & - & $0.89 \pm 0.02$ & $0.87 \pm 0.02$ \\
\hline$E_{\text {cut }}, \mathrm{keV}$ & - & - & $12.49 \pm 0.13$ & $12.93 \pm 0.25$ \\
\hline$E_{\text {fold }}, \mathrm{keV}$ & - & - & $11.10 \pm 0.18$ & $11.56 \pm 0.31$ \\
\hline$\tau_{\mathrm{cycl}}$ & - & $0.53_{-0.07}^{+0.08}$ & - & $0.16_{-0.04}^{+0.07}$ \\
\hline$E_{\text {cycl }}, \mathrm{keV}$ & - & $24.26 \pm 0.49$ & - & $23.2 \pm 1.0$ \\
\hline$\sigma_{\text {cycl }}, \mathrm{keV}$ & - & $5.93_{-0.42}^{+0.46}$ & - & $5.35_{-0.75}^{+1.13}$ \\
\hline$E_{\mathrm{Fe}}, \mathrm{keV}$ & $6.46 \pm 0.03$ & $6.45 \pm 0.03$ & $6.46 \pm 0.03$ & $6.46 \pm 0.03$ \\
\hline$\sigma_{\mathrm{Fe}}, \mathrm{keV}$ & 0.2 (fix) & 0.2 (fix) & 0.2 (fix) & 0.2 (fix) \\
\hline$E W, \mathrm{eV}$ & $106_{-11}^{+23}$ & $121_{-9}^{+29}$ & $117_{-13}^{+13}$ & $118_{-8}^{+12}$ \\
\hline Flux $(3-79 \mathrm{keV})$ & \multirow{2}{*}{\multicolumn{4}{|c|}{$\begin{array}{c}\left(1.695_{-0.016}^{+0.010}\right) \times 10^{-10} \mathrm{erg} \mathrm{s}^{-1} \mathrm{~cm}^{-2} \\
\left(3.107_{-0.029}^{+0.019}\right) \times 10^{36} \mathrm{erg} \mathrm{s}^{-1}\end{array}$}} \\
\hline Luminosity $(3-79 \mathrm{keV})^{\mathrm{b}}$ & & & & \\
\hline$\chi^{2}$ (d.o.f) & $1572.3(1127)$ & $1229.1(1124)$ & 1211.4(1127) & $1172.5(1124)$ \\
\hline
\end{tabular}

a the POWERLAW*HIGHCUT in the XSPEC package

b for the distance 12.4 kpc (Torrejón et al., 2010)

Residuals between the source spectrum and the COMPTT model are shown in Fig. 5b. In comparison with other models, the COMPTT model approximates the soft part of the spectrum quite well and has a clear physical meaning. At the same time, Fig. $5 \mathrm{~b}$ also shows a peculiarity in the spectrum - a deficit of photons around $20 \mathrm{keV}$, leading to a high $\chi^{2}$ value (Table 1$)$.

To investigate this feature, we added an absorption component to the model in the form of the GABS model in the XSPEC package. It led to a significant improvement of the fit quality and $\chi^{2}$ value (Table 1 ). Corresponding residuals are shown in Fig. 5c, the line centroid energy is $E_{\text {cycl }} \simeq 24.3 \mathrm{keV}$. It is important to note that some wavelike structure is still present in residuals, that can be connected both with physical reasons and also with imperfectness of the model used. We interpret this absorption feature as a possible cyclotron absorption line. Two models, GABS and CYCLABS in the XSPEC package, are usually used to approximate absorption lines. While both models are described them adequate, the cyclotron line energy derived from the GABS model is systematically higher by several keV than the energy derived from the CYCLABS model (see, e.g., Tsygankov, Krivonos, \& Lutovinov 2012; Lutovinov et al. 2015). For IGR J18027-2016 and the CYCLABS model, the cyclotron line energy will be $E_{\text {cycl }} \simeq 20.2$ $\mathrm{keV}$.

Besides the cyclotron absorption line, a strong emission feature associated with the fluorescent iron emission line is clearly visible near $6.4 \mathrm{keV}$. To take it into account, a corresponding component in the Gaussian form was added to the model. The final best fit parameters as well as the source intensity and luminosity are summarized in Table 1.

In order to trace the evolution of the source spectrum and its parameters over the pulse period, we carried out pulse phase-resolved spectroscopy. Taking into account the relative faintness of the source (its flux is only about 4-5 $\mathrm{mCrab}$ ), the spin period was divided into four wide phase bins, roughly corresponding to the main and secondary peaks (Fig. 6). Such a division allows us to obtain spectra with good statistical quality at each phase and to determine the spectral parameters.

To approximate pulse phase-resolved spectra, we used the same model as for the averaged spectrum. However, the temperature of the seed photons $k T_{0}$ and the cyclotron line width were fixed at the values measured for the averaged spectrum because the temperature and width are poorly constrained due to the limitations of the spectral quality of the spectra. The column density was also fixed because only NuSTAR data (i.e., $>3 \mathrm{keV}$ ) were used for the phaseresolved spectroscopy.

Variations of the spectral parameters over the pulse are shown in Fig. 6. For better visualization, the pulse profile of IGR J18027-2016 in a wide energy band 3-79 keV is plotted in each panel by the grey histogram. The continuum parameters $(k T$ and $\tau)$ change slightly with the pulse phase, demonstrating a possible anticorrelation between them. In contrast, the cyclotron line energy demonstrates strong dependence of the pulse phase - in the main peak, it is around $E_{\text {cycl }} \simeq 22 \mathrm{keV}$, while in the secondary peak, it increases to $E_{\text {cycl }} \simeq 28 \mathrm{keV}$. Taking into account a relatively small width of the line $(\sim 5.5 \mathrm{keV})$ it can lead to the wave-like structure in the residuals (see Fig. $5 \mathrm{c}$ ).

Finally, it is necessary to note that, formally, the best approximation of the IGR J18027-2016 spectrum is given when the POWERLAW*HIGHCUT model is used for the continuum. This model was proposed by White, Swank, \& Holt (1983) to approximate spectra of accreting X-ray pulsars. Despite the artificiality and presence of the abrupt break at the cutoff energy, it describes spectra obtained with the majority of X-ray observatories more or less adequately. The 
significant increase of the data quality in hard X-rays with the NUSTAR observatory raises a question about the physically based models for X-ray pulsar emission as, e.g., use of the POWERLAW*HIGHCUT model can lead to artificial narrow absorption lines near the cutoff energy. Nevertheless, the IGR J18027-2016 spectrum is an example where this model works and describes it without arising additional artificial features (see Table 1 ). The $\chi^{2}$ value for this model is significantly lower than for COMPTT and is comparable with COMPTT + GABS. However, even in this case, there is still some noticable deficit of photons around $20 \mathrm{keV}$, which can be approximated by the adding of the GABS component to the model (see Fig. 5d,e). The improvement of the fit quality for this case is not so significant as for the COMPTT model. Therefore, we performed extensive Monte-Carlo simulations $\left(10^{5}\right.$ trials $)$ to estimate the significance of the line detection and found that it is about $4 \sigma$ for the POWERLAW*HIGHCUT continuum model.

\section{DISCUSSION AND CONCLUSION}

In this paper, we report results of the $N u S T A R$ observations of the absorbed supergiant system IGR J18027-2016. They can be summarized as follows: 1) the system demonstrates approximately constant X-ray luminosity on a time scale of more than dozen years; 2) during this time interval, the pulsar spun down with a rate of $\left.\dot{P} \simeq 6 \times 10^{-10} \mathrm{~s} \mathrm{~s}^{-1} ; 3\right)$ the possible presence of a cyclotron absorption line at energy $\sim 23 \mathrm{keV}$ is found in the source X-ray spectrum.

The observed increase of the rotation period of the neutron star in IGR J18027-2016 can be explained in terms of the wind accretion model in a settling regime (Shakura et al. 2012, 2014), which can be realized onto slowly rotating magnetized neutron stars at X-ray luminosities below $4 \times 10^{36} \mathrm{erg} \mathrm{s}^{-1}$. The observed stable X-ray luminosity suggests that the X-ray pulsar has reached its equilibrium period, which, in this model, depends on the binary orbital period $P_{b}$, stellar wind velocity $v_{w}$, neutron star magnetic moment $\mu$ and mass accretion rate $\dot{M}$ :

$$
P_{e q} \simeq 940[\mathrm{~s}] \mu_{30}^{12 / 11}\left(\frac{P_{b}}{10 \mathrm{~d}}\right) \dot{M}_{16}^{-4 / 11} v_{8}^{4},
$$

where the characteristic parameters are $\mu_{30} \equiv$ $\mu / 10^{30}\left[\mathrm{G} \mathrm{cm}^{3}\right], \dot{M}_{16} \equiv \dot{M} / 10^{16}\left[\mathrm{~g} \mathrm{~s}^{-1}\right], v_{8} \equiv v_{w} / 10^{8}\left[\mathrm{~cm} \mathrm{~s}^{-1}\right]$. A very strong dependence on the wind velocity suggests that it is more reliable to estimate the wind velocity from using this formula:

$$
v_{8} \simeq 0.6 \dot{M}_{16}^{1 / 11} \mu_{30}^{-3 / 11}\left(\frac{P_{e q} / 100 \mathrm{~s}}{P_{b} / 10 \mathrm{~d}}\right)^{1 / 4} .
$$

Assuming that the absorption feature at $23 \mathrm{keV}$ is the cyclotron line, the neutron star dipole magnetic moment is $\mu_{30} \sim 2$ and from the X-ray luminosity (see Table 1 ), we find $\dot{M}_{16} \simeq 3$. Therefore, using $P_{e q}=139.6 \mathrm{~s}$ and $P_{b} \simeq 4.6 \mathrm{~d}$, from Eq.2, we can estimate the wind velocity, which very weakly depends on other parameters: $\dot{M}$ and $\mu, v_{8} \sim 0.7$. This rather low value is very close to the wind velocity measured in the prototypical persistent HMXB with $\mathrm{OB}$ su- pergiant Vela X-1 (Giménez-García et al. 2016), suggesting similarity between the two sources.

In the model of settling quasi-spherical accretion, the neutron star close to equilibrium can exhibit either spin-up or spin-down, depending on whether the actual $\dot{M}$ is above or below the critical mass accretion rate, $\dot{M}_{e q}$ (see Eq. (2) in Postnov et al. 2014). For the parameters of IGR J180272016, we find $\dot{M}_{e q} \simeq 3.6 \times 10^{16} \mathrm{~g} \mathrm{~s}^{-1}$, i.e. indeed spindown of the neutron star is possible in this system. The observed value of the negative torque acting on the neutron star in IGR J18027-2016 $\dot{\omega} \simeq 2 \times 10^{-13} \mathrm{rad} \mathrm{s}^{-2}$, does not exceed the maximum possible negative torque, $\omega_{s d, \text { max }} \simeq 10^{-12} \mathrm{rad} \mathrm{s}^{-2}$, for the parameters of IGR J180272016 (see Eq. (6) in Postnov et al. 2014). Thus, the observed steady-state spin-down is consistent with expectations from the settling accretion model.

As discussed in Section 4, the spectral analysis revealed the possible presence of a cyclotron absorption line in the spectrum of IGR J18027-2016 at energies 23-24 keV. Additional independent for the existence of this feature comes from the timing properties of the source. Namely, the pulse profile and pulsed fraction dependencies on the energy have prominent features near the same energy. Particularly, the relative intensity of two peaks in the profile changes around 22-24 keV (see Fig. 3). Such behaviour was shown to be typical for another well known X-ray pulsar V 0332+53 with a well established cyclotron feature (Tsygankov et al. 2006). The observed non-monotonic dependence of the pulsed fraction on energy (see Fig. 4) is also typical for pulsars with cyclotron lines (Lutovinov \& Tsygankov 2009; Ferrigno et al. 2009). These additional observational facts indirectly support the presence of the cyclotron absorption line in the IGR J18027-2016 spectrum despite of its low significance for the POWERLAW*HIGHCUT continuum model.

The measured X-ray luminosity in IGR J18027-2016, $L_{x} \simeq 3 \times 10^{36} \mathrm{erg} \mathrm{s}^{-1}$, suggests that accretion onto the neutron star occurs in the subcritical regime where the radiation plays a secondary role (see, e.g. Basko \& Sunyaev 1976; Mushtukov et al. 2015). In this case the accretion flow is decelerated in a collisionless shock formed at some height above the neutron star surface (Langer \& Rappaport 1982; Bykov \& Krasil'shchikov 2004). The formation of a cyclotron line downstream of the shock occurs in the resonance layer in the inhomogeneous magnetic field, so the line profile can be more complicated than the simple Dopplerbroadened line (Zheleznyakov 1996). For example, depending on the geometry, the line may have a flat bottom or show emission wings. The complex shape of the residuals shown in Fig. 5, which are obtained assuming a Gaussian form of the line, may suggest a complicated line profile or may be an evidence for the presence of the first vacuum cyclotron feature at energies below the fundamental cyclotron line energy. This interesting result should be definitely be studied in the future with deeper observations.

\section{ACKNOWLEDGMENTS}

This work was supported by the Russian Science Foundation (grant 14-22-00271). Work of PK is partially supported by RFBR grant 14-12-00657. The research has made use of data obtained with $N u S T A R$, a project led by Caltech, funded by 
NASA and managed by NASA/JPL, and has utilized the NUSTARDAS software package, jointly developed by the ASDC (Italy) and Caltech (USA).

\section{REFERENCES}

Anders E., Grevesse N., 1989, GeCoA, 53, 197

Augello G., Iaria R., Robba N. R., Di Salvo T., Burderi L., Lavagetto G., Stella L., 2003, ApJ, 596, L63

Basko M. M., Sunyaev R. A., 1976, MNRAS, 175, 395

Bodaghee A., et al., 2014, ApJ, 791, 68

Bodaghee A., et al., 2016, ApJ, 823, 146

Boldin P. A., Tsygankov S. S., Lutovinov A. A., 2013, AstL, 39, 375

Bykov A. M., Krasil'shchikov A. M., 2004, AstL, 30, 309

Falanga M., Bozzo E., Lutovinov A., Bonnet-Bidaud J. M., Fetisova Y., Puls J., 2015, A\&A, 577, A130

Ferrigno C., Becker P. A., Segreto A., Mineo T., Santangelo A., 2009, A\&A, 498, 825

Filippova E. V., Tsygankov S. S., Lutovinov A. A., Sunyaev R. A., 2005, AstL, 31, 729

Giménez-García A., et al., 2016, A\&A, 591, A26

Harrison F. A., et al., 2013, ApJ, 770, 103

Hill A. B., et al., 2005, A\&A, 439, 255

Krivonos R. A., et al., 2014, ApJ, 781, 107

Langer S. H., Rappaport S., 1982, ApJ, 257, 733

Lutovinov A., Revnivtsev M., Molkov S., Sunyaev R., 2005, A\&A, 430, 997

Lutovinov A. A., Tsygankov S. S., 2009, AstL, 35, 433

Lutovinov A. A., Tsygankov S. S., Suleimanov V. F., Mushtukov A. A., Doroshenko V., Nagirner D. I., Poutanen J., 2015, MNRAS, 448, 2175

Madsen K. K., et al., 2015, ApJS, 220, 8

Mori K., et al., 2015, ApJ, 814, 94

Mason, A. B., Norton, A. J., Clark, J. S., Negueruela, I., \& Roche, P. 2011, A\&A, 532, A124

Mushtukov A. A., Suleimanov V. F., Tsygankov S. S., Poutanen J., 2015, MNRAS, 447, 1847

Postnov K. A., Shakura N. I., Kochetkova A. Y., Hjalmarsdotter L., 2014, EPJWC, 64, 02002

Revnivtsev M. G., et al., 2004, AstL, 30, 382

Shakura N., Postnov K., Kochetkova A., Hjalmarsdotter L., 2012, MNRAS, 420, 216

Shakura N. I., Postnov K. A., Kochetkova A. Y., Hjalmarsdotter L., 2014, EPJWC, 64, 02001

Shtykovsky A.E., Tsygankov S.S., Lutovinov A.A. and Molkov S.V., Astronomy Letters, in press (2016)

Torrejón J. M., Negueruela I., Smith D. M., Harrison T. E., 2010, A\&A, 510, A61

Tsygankov S. S., Lutovinov A. A., Churazov E. M., Sunyaev R. A., 2006, MNRAS, 371, 19

Tsygankov S. S., Krivonos R. A., Lutovinov A. A., 2012, MNRAS, 421, 2407

Tsygankov S. S., Lutovinov A.A., Krivonos R.A., Molkov S.V., Jenke P.J., Finger M.H., Poutanen J., 2016, MNRAS, 457, 258

Walter R., Lutovinov A. A., Bozzo E., Tsygankov S. S., 2015, A\&ARv, 23, 2

White N. E., Swank J. H., Holt S. S., 1983, ApJ, 270, 711

Wik D. R., et al., 2014, ApJ, 792, 48

Zheleznyakov V. V., 1996, ASSL, 204,

This paper has been typeset from a $\mathrm{T}_{\mathrm{E} X} / \mathrm{LAT}_{\mathrm{E}} \mathrm{X}$ file prepared by the author. 